\title{
Performance studies of large-area triple-GEM prototypes for future upgrades of the CMS forward muon system
}

Salvatore A. TUPPUTI*, M. Abbrescia, A. Colaleo, G. de Robertis, F. Loddo, M. Maggi, S. Nuzzo

Politecnico di Bari and INFN Sezione di Bari - Bari, Italy

E-mail: salvatore.tupputi@cern.ch

Y. Ban, J. Cai, H. Teng

Peking University - Beijing, China

A. Mohapatra, T. Moulik

NISER - Bhubaneswar, India

A. Gutierrez, P. E. Karchin

Wayne State University - Detroit, USA

L. Benussi, S. Bianco, S. Colafranceschi, D. Piccolo, G. Raffone, G. Saviano Labortori Nazionali di Frascati INFN - Frascati, Italy

D. Abbaneo, P. Aspell, S. Bally, U. Berzano, J. Bos, J. P. Chatelain, J. Christiansen, A. Conde Garcia, E. David, R. De Oliveira, S. Duarte Pinto, S. Ferry, F. Formenti, A. Marchioro, H. Postema, A. Rodrigues, L. Ropelewski, A. Sharma, N. Smilkjovic, M. Zientek

Physics Department, CERN - Geneva, Switzerland

A. Marinov, M. Tytgat, N. Zaganidis

Department of Physics and Astronomy Universiteit Gent - Gent, Belgium

K. Gnanvo, M. Hohlmann, M. J. Staib

Florida Institute of Technology - Melbourne, USA

G. Magazzu, E. Olivieri, N. Turini

INFN Sezione di Pisa - Pisa, Italy

K. Bunkowski

Warsaw University - Warsaw, Poland 


\begin{abstract}
The RPC muon system of the CMS detector at the CERN LHC remains uninstrumented in the pseudorapidity region $1.6<|\eta|<2.4$. An ongoing project aims at covering the region of the muon endcaps with large-area triple-GEM detectors whose features are suited to enhance muon tracking and preserve triggering capabilities for the CMS detector upgrade. The design and assembling of small $(10 \mathrm{~cm} \times 10 \mathrm{~cm})$ and full-size trapezoidal $(1 \mathrm{~m} \times 0.5 \mathrm{~m})$ triple-GEM prototypes will be described. The prototypes have been tested with soft $\mathrm{x}$-rays and with a pion/muon test beam at the CERN SPS. Results from measurements with different experimental set-up on detector resolution and efficiency as well as timing performance will be reported. Preliminary simulation results will be discussed, related to studies on performance variations of the expected muon tracks reconstruction and trigger performance for different upgraded muon system scenarios.
\end{abstract}

The 2011 Europhysics Conference on High Energy Physics, EPS-HEP 2011,

July 21-27, 2011

Grenoble, Rhône-Alpes, France

${ }^{*}$ Speaker. 


\section{GEM for CMS}

The muon system of Compact Muon Solenoid (CMS) [1] experiment at the CERN Large Hadron Collider (LHC) employs Resistive Plate Chambers (RPC) whose time resolution $(\sim n s)$ assigns the correct bunch crossing in Level-1 muon trigger. They are double-gap $\phi$-strip readout bakelite RPCs operated in avalanche mode with a $\mathrm{C}_{2} \mathrm{H}_{2} \mathrm{~F}_{4}: \mathrm{iC}_{4} \mathrm{H}_{10}: \mathrm{SF}_{6}$ (94.7:5:0.3) mixture. The current RPC system covers the $|\eta|<1.6$ pseudorapidity region; an extension up to $|\eta|<2.1$ is suitable for the particle flux $(\sim 10 \mathrm{kHz})$ expected with the LHC luminosity upgrade $\left(L \sim 10^{34-35} \mathrm{~cm}^{-2} \mathrm{~s}^{-1}\right)$. In such scenario the RPCs radiation hardness is at the edge while large strips pitch may affect high rate system performance, even with adequate time resolution. Micro-pattern gaseous detectors (MPGD) can reach rate capabilities up to $10 \mathrm{MHz} / \mathrm{cm}^{2}$ and high spatial $(\sim 100 \mu \mathrm{m})$ and time $(\lesssim 5 \mathrm{~ns})$ resolutions, with a $\sim 98 \%$ detection efficiency. They can be operated with non-flammable gas mixtures and finer readout granularity along both $\eta$ and $\phi$ allows for either triggering or tracking. A dedicated R\&D program has chosen to pursue the Gas Electron Multipliers (GEM) technology [2].

\section{Simulation studies results}

Monte Carlo single muon samples have been generated (with PYTHIA [3]) and CMS detector simulated (with GEANT4 [4]) with a RPC system extending up to $|\eta|<2.1$, whereas the first two RPC stations in $1.6<|\eta|<2.1$ have been upgraded in spatial resolution. The strips pitch of these RPCs has been set to several widths, each corresponding to a given GEM spatial resolution. Each width is accounted for as multiple of the resolution given by nominal (CMS TDR [5]) RPCs strips width $(\sim 2 \mathrm{~cm})$, see table 1 . The muons transverse momentum can be $0.2,0.5,1,2,5 \mathrm{TeV} / \mathrm{c}$.

\begin{tabular}{|c|c|c|c|c|c|c|c|c|}
\hline \hline Resolution & TDRx1 & TDRx2 & TDRx4 & TDRx8 & TDRx16 & TDRx32 & TDRx64 & TDRx128 \\
\hline Trigger & TDRx1 & TDRx2 & & TDRx8 & & & & \\
\hline \hline
\end{tabular}

Table 1: Simulated resolutions for the GEM-like RPCs. Only three setups have been used for trigger studies.

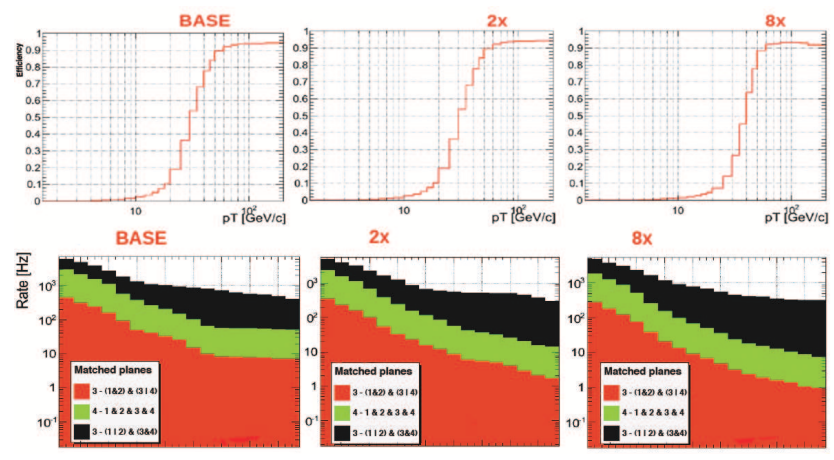

Figure 1: Up: Trigger turn-on curves for $p_{T}>50 \mathrm{GeV} / \mathrm{c}$ threshold. Down: Trigger rates vs $p_{T}$ thresholds.

\subsection{Trigger capability}

The CMS RPC muon trigger algorithm [6] compares RPC hits in the same bunch crossing, in at least 3 stations, with preloaded patterns assigning the muon candidate $\mathrm{p}_{T}, \eta$ and $\phi$. Assigned 

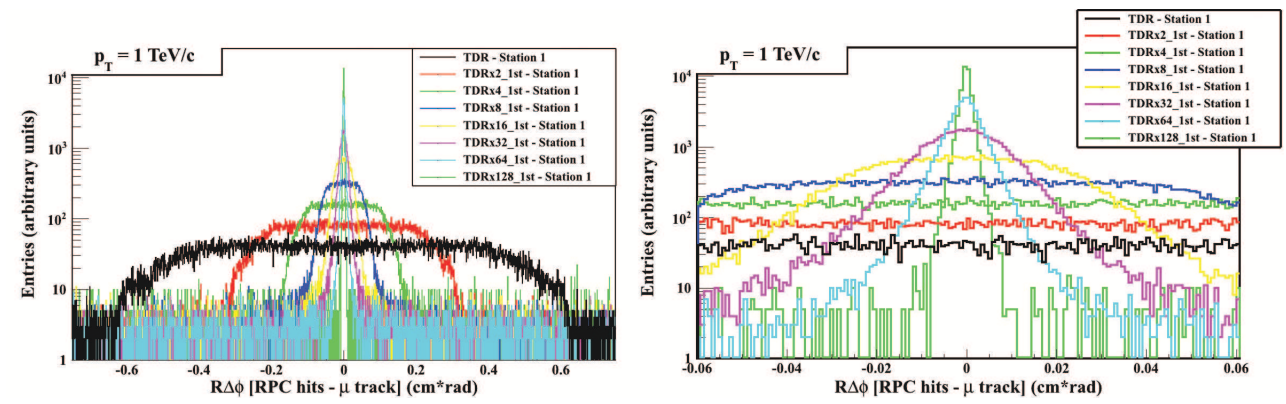

Figure 2: Global (left) and zoomed (right) spatial residuals distributions comparison for $p_{T}=1 \mathrm{TeV} / \mathrm{c}$ muons.

momentum precision depends on spatial granularity along $\phi$, hence an improved spatial resolution sharpens the turn-on curves and lowers the rates. Fig. 1 shows that for a $p_{T}>50 \mathrm{GeV} / \mathrm{c}$ threshold the turn-on slope steepness increases with resolution and that single muon rate in the thresholds region $p_{T} \gtrsim 25 \mathrm{GeV} / \mathrm{c}$ is flat for TDR, while it decreases with $p_{T}$ for higher resolutions.

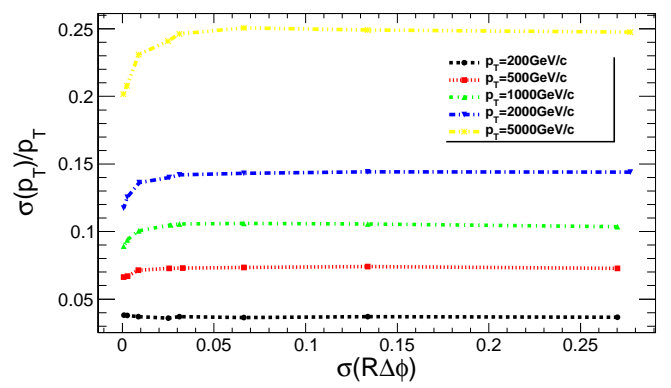

Figure 3: Momentum resolution vs spatial resolution for different muon momenta.

\subsection{Muon tracks reconstruction}

The spatial resolution lower limit, set by the electromagnetic processes (mainly multiple scattering) muon experiences in traversing muon system material, has been estimated from simulation to be $\sim 100 \mu \mathrm{m}$ for a $p_{T}=1 \mathrm{TeV} / \mathrm{c}$ muon. In fig. 2 single muons spatial residuals, from which resolution is obtained, for different strip widths are compared. The strips width dominates the resolution down to widths a factor 8 more narrow than the TDR ones. From a factor 16 upward $(\sigma \sim 200 \mu \mathrm{m})$ the distributions approach a gaussian shape. Tracks reconstructed in the CMS muon system (stand-alone muons) have been used to estimate transverse momentum resolution, provided they are selected by the CMS global muon algorithm [7]. Transverse momentum resolution versus the corresponding spatial resolution (fig. 3) shows that the momentum resolution starts improving as spatial resolution turns from uniform to gaussian.

\section{Test beam results on first prototypes}

The first experimental tests [2] have been conducted on $10 \times 10 \mathrm{~cm}^{2}$ triple-GEM prototypes. 


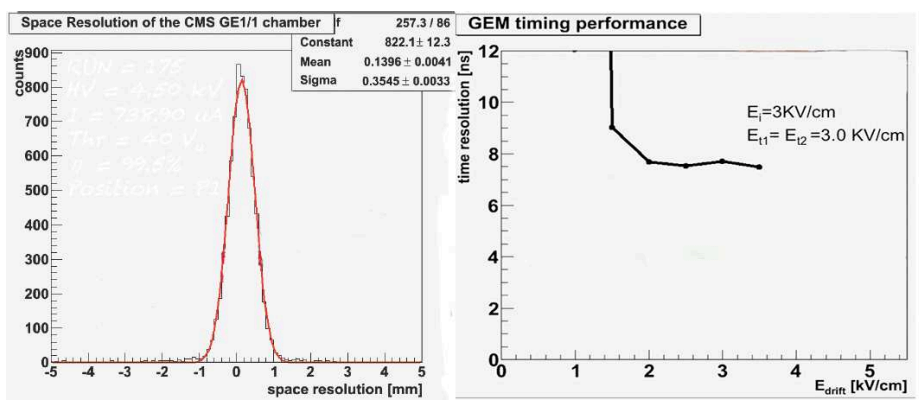

Figure 4: Spatial (left) and time (right) resolutions from test beam.

The eventual detector will be large scaled, hence advanced building technologies like single-mask etching [8], which improves the alignment, and auto-stretching, which allows for better operational stability and gain performance, have been employed. The largest triple-GEM prototype ever built has an active area of $990 \times(220-445) \mathrm{mm}^{2}, 3072$ read-out channels and a strip pitch of $0.8 \mathrm{~mm}$. The electronics read-out relies on electronic boards based on VFAT chips [9], with a 128 analog channels front-end able to provide binary output for tracking and to be ORed for triggering. The internal clock can sample a $40 \mathrm{MHz}$ signal. A test beam with pions has taken place in October 2010 with chambers gaps spacing of 3/2/2/2 mm and a $\mathrm{Ar}: \mathrm{CO}_{2}$ (70:30) gas mixture. A space resolution of $\sim 300 \mu \mathrm{m}$ and a time resolution of $\lesssim 8 \mathrm{~ns}$ have been measured (fig. 4). After encouraging tests on small prototypes, another test beam took place in July 2011 on a large scale prototype with 3/1/2/1 $\mathrm{mm}$ gaps spacing and a $\mathrm{Ar}: \mathrm{CO}_{2}: \mathrm{CF}_{4}$ gas mixture, whose data are being processed.

\section{Conclusions}

Simulations prove that a CMS RPC system upgrade with GEMs would bring relevant improvements to triggering and tracking capabilities. Tests on triple-GEM prototypes have shown time resolution is comparable with the RPCs one while triple-GEMs spatial resolution sits on the turning point from uniform to gaussian behavior (fig. 2). Physics processes simulations for assessing GEMs contribution to improve CMS physics reach and further test beams are the next steps.

\section{References}

[1] CMS Coll., The CMS muon project: Technical Design Report, CERN-LHCC-97-032.

[2] M. Tytgat et al., Characterization of GEM Detectors [...], 10.1109/NSSMIC.2010.5874006.

[3] T. Sjostrand et al. High Energy Physics event generator with PYTHIA 6.1, arXiv hep-ph/0010017.

[4] S. Agostinelli et al. Geant4 - A Simulation Toolkit, Nucl. Instr. and Methods A 506 (2003) 250.

[5] CMS Coll., Physics TDR, Volume I, Detector Performance and Software, CERN-LHCC-2006-001.

[6] CMS Coll., The trigger and Data Acquisition project, Volume I, CERN-LHCC-2000-038.

[7] G. Abbiendi et al., Muon Reconstruction in CMS Detector, CMS AN-2008/097.

[8] M. Villa et al., Nucl. Instr. and Methods A, 628 (2011) 182.

[9] P. Aspell et al., VFAT2: A front-end system on chip [...], TWEPP 2007 Proceedings. 